\title{
Inclusão Digital: um mapeamento sistemático de ferramentas e aplicativos para pessoas com deficiência
}

Mayara Benício de Barros Souza - Universidade Federal do Vale do São Francisco (UNIVASF) - mayara.benicio@univasf.edu.br

Walla Nascimento Souza - Universidade Federal do Vale do São Francisco (UNIVASF)wallasousa50@gmail.com

Resumo: Este artigo busca identificar ferramentas e aplicativos para a inclusão digital de pessoas com deficiência. Para isto, foi realizado um mapeamento sistemático para coletar e identificar essas informações. Esse mapeamento retornou inicialmente 339 estudos, dos quais foram selecionados 27 como estudos primários, possibilitando extrair 35 ferramentas e aplicativos, e 12 características funcionais deles. Essa quantidade de ferramentas e aplicativos obtidas desse mapeamento pode ser considerada relevante, o que possibilita inferir sobre a importância da utilização desses meios para a inclusão digital dos indivíduos portadores de algum tipo de deficiência.

Palavras-chave: mapeamento sistemático, inclusão digital, pessoas com deficiência, ferramentas, aplicativos.

Abstract: This article seeks to identify tools and applications for digital inclusion of people with disabilities. For this, a systematic mapping was performed, to collect and identify this information. This mapping initially returned 339 studies, of which 27 were selected as primary studies, allowing to extract 35 tools and applications, and 12 functional characteristics of them. This amount of tools and applications obtained from this mapping can be considered relevant, which makes it possible to infer about the importance of using these means for the digital inclusion of individuals with disabilities. Keywords: systematic mapping, digital inclusion, people with disabilities, tools, applications.

\section{Introdução}

Ao longo da história a revolução tecnológica trouxe uma forma mais ágil e eficaz para que os indivíduos pudessem exercer suas atividades. Porém, muitos ainda não se adaptaram a esta nova realidade, visto que se faz necessário o conhecimento das ferramentas digitais e como utilizá-las em favor de sua interação no cotidiano (Victor, Drago e Chicon, 2013). Neste contexto a inclusão digital ganha notoriedade, pois trata da utilização pelas pessoas das mais diversas ferramentas digitais, com o intuito de integrar o indivíduo a tecnologia. Essa inclusão tem também o objetivo de buscar métodos que viabilizem o desenvolvimento tecnológico para que as inovações estejam acessíveis a todos. É mister ressaltar que a inclusão digital não é restrita apenas ao acesso das pessoas as tecnologias, mas também ao devido conhecimento sobre suas utilidades. Desse modo, os indivíduos ao se tornarem conhecedores das tecnologias, serão capazes de se aperfeiçoar para o mercado de trabalho. Assim, a acessibilidade digital dará mais oportunidades as pessoas mais vulneráveis, dando-lhes condições de inserção no mercado de trabalho (Cruz, 2004; Medeiros e Carvalho, 2006).

V. $18 \mathrm{~N}^{\mathrm{o}} 1$, julho, 2020 DOI: 
Uma parcela da população ainda mais vulnerável é a de pessoas com deficiência. As limitações relacionadas a elas podem torna-se inexistentes, quando há um trabalhado deste tipo de inclusão digital com o propósito de qualificação e preparo. Isto dará a estes indivíduos uma maior autonomia perante a sociedade (Nazareno et. al., 2006). Cabe ressaltar que o acesso a tecnologias assistivas é um direito assegurado pela Lei $\mathrm{N}^{\mathrm{o}}$ 13.146, a Lei Brasileira de Inclusão (LBI), no qual todos os 3 poderes do executivo devem promover a inserção plena das pessoas com algum tipo de deficiência na sociedade (Brasil, 2015). Diante disto, este artigo tem o objetivo de apresentar um mapeamento sobre ferramentas e aplicativos utilizados para inclusão digital que podem ser utilizados por pessoas com algum tipo de deficiência.

\section{Material e métodos}

Nesta pesquisa foi utilizado o mapeamento sistemático usualmente desenvolvido em pesquisas de engenharia de software que permite examinar e conhecer os estudos publicados e, classificá-los por meio de síntese e mapeamento dos resultados. Essa metodologia permite uma visão geral sobre o assunto pesquisado, utilizando-se dos seguintes passos: definição de questões de pesquisa, realização de buscas em estudos relevantes, seleção dos estudos por meio de palavras-chaves e resumo, extração dos dados e mapeamento (Kitchenham e Charters, 2007; Petersen et. al., 2007). Como este estudo tem o objetivo de identificar ferramentas e aplicativos utilizados para inclusão digital de pessoas com algum tipo de deficiência, a pesquisa partiu dos seguintes questionamentos:

- Quais as ferramentas e aplicativos mais utilizados para a inclusão digital de pessoas com deficiência?

- Quais são as principais funcionalidades, para os usuários, das ferramentas e aplicativos voltados para inclusão digital?

Para determinar quais seriam os termos de busca utilizados para responder as questões de pesquisa, foi utilizada a seguinte estratégia: i) identificação das palavraschaves, ii) identificação de sinônimos, iii) tradução para o inglês das palavras-chaves e iv) geração das strings de busca. De acordo com estes passos foi definida as strings de busca (ver Quadro 1) que serão aplicadas nos repositórios de estudos científicos. É válido ressaltar que essa string pode sofrer adaptações mínimas, devido à técnica de estratégia de busca utilizada pelos repositórios na sua execução.

Quadro 1. Strings de busca.

\begin{tabular}{|l|}
\hline \multicolumn{1}{|c|}{ Strings de busca } \\
\hline $\begin{array}{l}\text { (("inclusão digital") OR ("inclusão social" AND "tecnologia da informação")) AND ("ferramenta" OR } \\
\text { "software" OR "aplicativos") AND ("deficiência" OR "deficiente" OR "deficit" OR "incapacidade" ) }\end{array}$ \\
\hline $\begin{array}{l}\text { (("digital inclusion") OR ("social inclusion" AND "information technology")) AND ("tool" OR } \\
\text { "software" OR "applications") AND ("disability" OR "disabled" OR "deficit" OR "inability") }\end{array}$ \\
\hline
\end{tabular}

Fonte: Elaborado pelos autores (2019). 
Os repositórios de estudos científicos selecionados para a busca dos estudos primários, que disponibilizaram mecanismos de consultas avançados e retornaram um número de estudos satisfatórios, foram:

- Google Scholar (Disponível em: https://scholar.google.com.br);

- RENOTE - Revista Novas Tecnologias na Educação (Disponível em: https:// seer.ufrgs.br/renote);

- ACM Digital Library (Disponível em: http://portal.acm.org);

- BDTD - Biblioteca Digital Brasileira de Teses e Dissertações (Disponível em: http://bdtd.ibict.br/).

Os trabalhos incluídos para o estudo deste mapeamento foram selecionados pelos seguintes critérios: estarem disponíveis de forma online, possuírem embasamento científico e terem o conteúdo totalmente acessível. Foram excluídos os estudos repetidos, os que não estavam adequados ao contexto da pesquisa ou apresentavam conteúdo com restrição de acesso. Os passos seguidos para seleção dos estudos primários incluiram: execução da strings de busca definida no mecanismo de busca; avaliação dos estudos retornados de acordo com o título e palavras-chaves; e leitura dos resumos que possibilitaram a coleta dos Estudos Primários (EPs), que foram lidos na íntegra.

\section{Resultados e discussão}

A partir das strings e dos repositórios de buscas selecionados, um total 339 estudos foram retornados. O mecanismo que retornou mais artigos foi o Google Scholar com 163 estudos. Em seguida o Renote com 81 estudos. Os dois mecanismos que retornaram menos estudos foram o ACM com 67 estudos e a BDTD com 28. O Gráfico 1 abaixo retrata esses resultados para cada mecanismo de busca utilizado. Para a busca dos EPs foi delimitado o ano de suas publicações entre os períodos de 2000 à 2019, com o intuito de retornar dados mais atuais.

Gráfico 1.Número de estudos retornados.

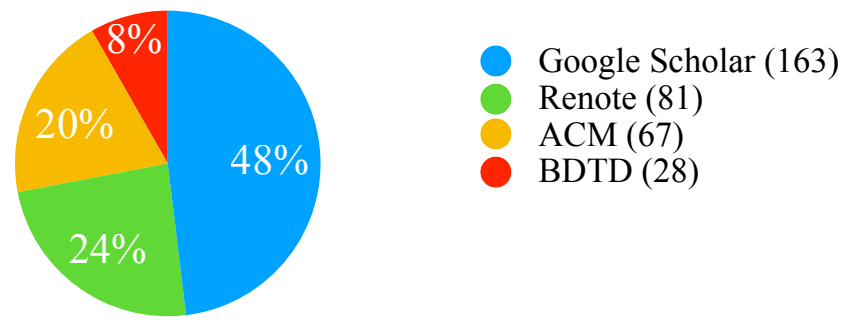

Fonte: Elaborado pelos autores (2019).

A partir do Gráfico 1 pode-se verificar que o mecanismo de busca Google Scholar obteve quase metade dos estudos retornados (48\%). Pode-se inferir que isto se deu, pois este repositório coleta dados de outras publicações online de conteúdo científico. O segundo repositório que mais retornou estudos, foi a revista Renote com 
24\%. Esse percentual é extremante significativo, visto que diferente do Google Scholar, ela retorna apenas artigos publicados especificamente no seu repositório. Depois desta revista, o repositório que mais retornou estudos foi a revista ACM com 20\%. Já a BDTD retornou a menor quantidade de estudos, apenas $8 \%$ do total. Baseado nestes estudos retornados, os métodos de seleção foram aplicados. O Quadro 2 abaixo exibe os resultados obtidos através dos procedimentos de seleção que foram descritos anteriormente. Com os estudos resultantes dessa seleção houve a leitura integral dos estudos incluídos. Estes foram considerados como EPs e foram fichados em um formulário. Ao final da primeira seleção, resultaram-se 75 estudos potencialmente relevantes para a pesquisa. A segunda seleção identificou 27 estudos que foram incluídos como EPs e 48 foram descartados.

Quadro 2. Seleção dos estudos primários.

\begin{tabular}{|c|c|c|c|c|c|c|c|}
\hline \multicolumn{8}{|c|}{ SELEÇÕES DE ESTUdOS PRIMÁRIOS } \\
\hline \multirow{3}{*}{ FONTE } & \multirow[b]{3}{*}{$\begin{array}{l}\text { ESTUDOS } \\
\text { RETORNA- } \\
\text { DOS }\end{array}$} & \multicolumn{2}{|c|}{$\begin{array}{c}1^{\mathrm{a}} \text { SELEÇÃO } \\
\text { (ESTUDOS } \\
\text { POTENCIALMENTE } \\
\text { RELEVANTES) }\end{array}$} & \multicolumn{4}{|c|}{$2^{\mathrm{a}}$ SELEÇÃO (INTRODUÇÃO/CONCLUSÃO) } \\
\hline & & \multirow[b]{2}{*}{$\begin{array}{l}\text { Título/ } \\
\text { PALAVRAS- } \\
\text { CHAVES }\end{array}$} & \multirow[b]{2}{*}{ RESUMO } & \multicolumn{3}{|c|}{ ExCLUídos } & \multirow{2}{*}{$\begin{array}{c}\text { INCLUÍDOS } \\
\text { ESTUDOS } \\
\text { PRIMÁRIOS }\end{array}$} \\
\hline & & & & $\begin{array}{c}\text { NÃO } \\
\text { RELEVANTE }\end{array}$ & $\begin{array}{l}\text { REPETIDO/ } \\
\text { DUPLICA- } \\
\text { DO }\end{array}$ & $\begin{array}{c}\text { INCOMPLE- } \\
\text { TO }\end{array}$ & \\
\hline $\begin{array}{l}\text { Google } \\
\text { Scholar }\end{array}$ & 163 & 78 & 35 & 19 & 1 & - & 15 \\
\hline Renote & 81 & 51 & 27 & 19 & - & - & 8 \\
\hline $\mathrm{ACM}$ & 67 & 25 & 8 & 5 & - & - & 3 \\
\hline BDTD & 28 & 13 & 5 & 3 & 1 & - & 1 \\
\hline Total & 339 & 167 & 75 & 46 & 2 & & 27 \\
\hline
\end{tabular}

Fonte: Elaborado pelos autores (2019).

Tomando-se como base o Quadro 2 acima, o Google Scholar manteve a alta representatividade no EPs, 15 estudos (56\%). A menor representatividade dos EPs ficou com o repositório BDTD, 1 estudo (4\%). O segundo mecanismo que mais retornou EPs foi a revista Renote, 8 estudos (30\%) e em seguida a ACM, 3 estudos (11\%). Após a finalização da seleção dos EPs, esses foram organizados com o seu respectivo identificador e a fonte da qual foi extraído. O Quadro 3 abaixo apresenta esses EPs selecionados e sua identificação. Os EPs 1, 7, 9, 14, 18, 19, 21, 23 e 25 foram aplicados com o público-alvo e os outros são estudos teóricos, sem participantes. 
Quadro 3. Identificação dos estudos primários.

\begin{tabular}{|c|c|c|c|}
\hline EP_ID & Ano & Estudo & Fonte \\
\hline EP_01 & 2018 & $\begin{array}{l}\text { Reconectando: Um estudo sobre o cego adquirido e a utilização de serious } \\
\text { games na sua reabilitação. }\end{array}$ & Google Scholar \\
\hline EP_02 & 2017 & $\begin{array}{l}\text { Q-LIBRAS: Um jogo educacional que usa libras para estimular alunos } \\
\text { surdos a aprenderem química. }\end{array}$ & Google Scholar \\
\hline EP_03 & 2015 & $\begin{array}{l}\text { A tecnologia como meio de inclusão dos deficientes visuais no transporte } \\
\text { público. }\end{array}$ & Google Scholar \\
\hline EP_04 & 2017 & $\begin{array}{l}\text { Análise de aplicativos para comunicação aumentativa e alternativa a } \\
\text { indivíduos com transtorno do espectro autista. }\end{array}$ & Google Scholar \\
\hline EP_05 & 2018 & $\begin{array}{l}\text { Utilizando a API google places para desenvolver uma solução de } \\
\text { mapeamento de locais com acessibilidade para pessoas com mobilidade } \\
\text { reduzida. }\end{array}$ & Google Scholar \\
\hline EP_06 & 2018 & $\begin{array}{l}\text { Protótipo de aplicativo para dispositivos móveis para mapeamento de } \\
\text { ambientes com acessibilidade arquitetônica: estudo de caso na cidade de } \\
\text { Marabá-PA. }\end{array}$ & Google Scholar \\
\hline EP_07 & 2013 & $\begin{array}{l}\text { Tecnologias Assistivas Livres para Inclusão de Alunos com Deficiência no } \\
\text { Projeto UCA. }\end{array}$ & Google Scholar \\
\hline EP_08 & 2014 & $\begin{array}{l}\text { Aplicativos de tradução para Libras e a busca pela validade social da } \\
\text { Tecnologia Assistiva. }\end{array}$ & Google Scholar \\
\hline EP_09 & 2017 & $\begin{array}{l}\text { Avaliação de Usabilidade do Aplicativo VLibras-Móvel com Usuários } \\
\text { Surdos. }\end{array}$ & Google Scholar \\
\hline EP_10 & 2019 & Para todos verem: recurso para integração e inclusão. & Google Scholar \\
\hline EP_11 & 2015 & $\begin{array}{l}\text { Estudo de Aplicativos Móveis para Deficientes Visuais no Âmbito } \\
\text { Acadêmico. }\end{array}$ & Google Scholar \\
\hline EP_12 & 2016 & $\begin{array}{l}\text { Competências Digitais e Segurança na Internet: informativo e orientações } \\
\text { para pais, professores e estudantes. }\end{array}$ & Google Scholar \\
\hline $\mathrm{EP} \_13$ & 2018 & $\begin{array}{l}\text { Produção científica brasileira sobre tradução automática português } \\
\text { brasileiro-libras: uma revisão sistemática de literatura. }\end{array}$ & Google Scholar \\
\hline $\mathrm{EP} \_14$ & 2018 & $\begin{array}{l}\text { Libras Game: trabalhando o ensino da matemática com alunos surdos dos } \\
\text { anos iniciais através do uso de aplicativo educacional. }\end{array}$ & Google Scholar \\
\hline $\mathrm{EP} \_15$ & 2016 & $\begin{array}{l}\text { Design para acessibilidade:inclusão de pessoas com deficiência visual ao } \\
\text { serviço de cinema. }\end{array}$ & Google Scholar \\
\hline $\mathrm{EP} \_16$ & 2014 & $\begin{array}{l}\text { Tecnologia Assistiva: a inserção de aplicativos de tradução na promoção de } \\
\text { uma melhor comunicação entre surdos e ouvintes. }\end{array}$ & RENOTE \\
\hline EP_17 & 2017 & $\begin{array}{l}\text { Aplicativos de Tradução Português-Libras na Educação Bilíngue: desafios } \\
\text { frente à desambiguação. }\end{array}$ & RENOTE \\
\hline EP_18 & 2017 & $\begin{array}{l}\text { Envelhecer@Saudável: um material educacional digital voltado ao público } \\
\text { idoso. }\end{array}$ & RENOTE \\
\hline EP_19 & 2016 & $\begin{array}{l}\text { Avaliação de um jogo sério digital destinado ao público idoso utilizando o } \\
\text { método gameflow. }\end{array}$ & RENOTE \\
\hline EP_20 & 2004 & $\begin{array}{l}\text { EDUKITO: propiciando a inclusão digital de Pessoas com Necessidades } \\
\text { Educacionais Especiais. }\end{array}$ & RENOTE \\
\hline EP_21 & 2014 & $\begin{array}{l}\text { Avaliação de tecnologias de tradução português-libras visando o uso no } \\
\text { ensino de crianças surdas. }\end{array}$ & RENOTE \\
\hline EP_22 & 2011 & $\begin{array}{l}\text { Matraca: Ferramenta Computacional para Auxílio à Inclusão Digital de } \\
\text { Deficientes Visuais. }\end{array}$ & RENOTE \\
\hline EP_23 & 2010 & Experiência de inclusão digital com deficiente visual. & RENOTE \\
\hline
\end{tabular}




\begin{tabular}{|l|l|l|l|}
\hline EP_24 & 2010 & $\begin{array}{l}\text { Fostering Digital Inclusion and Accessibility: The PorSimples project for } \\
\text { Simplification of Portuguese Texts. }\end{array}$ & ACM \\
\hline EP_25 & 2018 & $\begin{array}{l}\text { The Brazilian Challenge to Accessibility and Digital Inclusion for People } \\
\text { With Autistic Spectrum Disorders. }\end{array}$ & ACM \\
\hline EP_26 & 2017 & Accessibility and Affectibility in CPqD. & ACM \\
\hline EP_27 & 2015 & A utilização de aplicativos na alfabetização de surdos. & BDTD \\
\hline
\end{tabular}

Fonte: Elaborado pelos autores (2019).

Baseado no Quadro 3, observa-se que o ano de 2017 e 2018 foram os que mais retornaram EPs, 6 estudos cada. Seguido pelos anos de 2014, 2015 e 2016, com 3 estudos cada um. Os anos que retornaram menos estudos foram os de 2010 (2 estudos) e 2004, 2011, 2013 e 2019 (apenas 1 estudo cada). Com a análise dos EPs selecionados nesta pesquisa, foi possível obter evidências de 35 ferramentas e aplicativos e, 12 características com as principais funcionalidades delas. Essas evidências brutas estão representadas no Gráfico 2 que apresenta para cada EP a quantidade de ferramentas e aplicativos extraídos e a sua quantidade de características. Observa-se com este gráfico que o estudo EP_13 possibilitou a maior extração de ferramentas e aplicativos, 10 no total, seguido do estudo EP_12, com 7 ferramentas e aplicativos coletados.

Gráfico 2. Número de ferramentas e aplicativos e, características para cada estudo primário.

Ferramenta/Aplicativo

Características

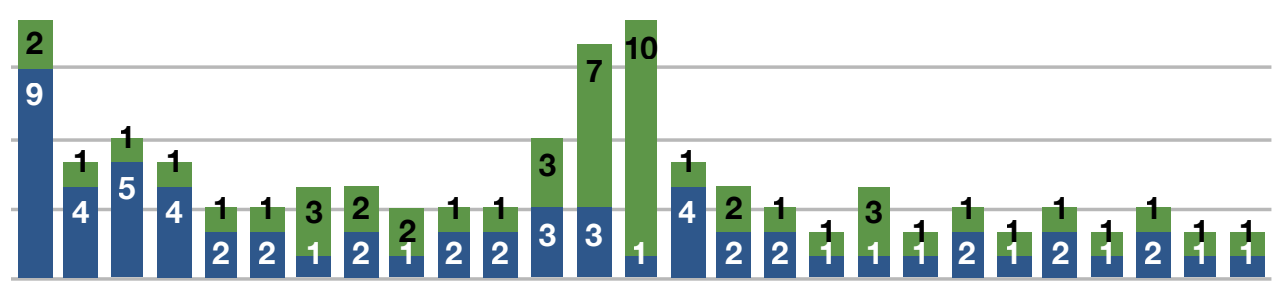

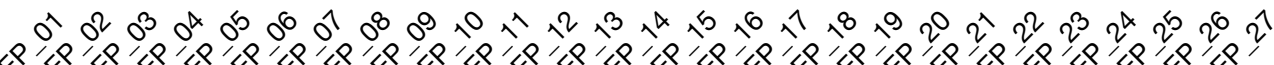

Fonte: Elaborado pelos autores (2019).

Para relacionar as ferramentas e aplicativos que foram extraídos dos EPs, as suas funcionalidades gerais foram caracterizadas no Quadro 4 abaixo, juntamente com um identificador. Para relacionar as ferramentas e aplicativos que foram extraídos dos estudos primários com os dados do Quadro 4 foi gerada a Tabela 1. Nesta tabela também cada ferramenta ou aplicativo recebeu: um identificador único associado a deficiência atendida; EPs utilizados para sua extração; e identificação se a tecnologia foi Desenvolvida no EP (D_EP) ou se é uma tecnologia Assistiva Clássica (AC). 
Quadro 4. Descrição das funcionalidades das ferramentas e aplicativos.

\begin{tabular}{|l|l|}
\hline $\begin{array}{c}\text { Identificador da } \\
\text { Funcionalidade } \\
\text { Aplicativo }\end{array}$ & \multicolumn{1}{|c|}{ Descrição da funcionalidade da Ferramenta/Aplicativo } \\
\hline FFA_01 & Descreve o ambiente em que o usuário está, através de um sistema colaborativo. \\
\hline FFA_02 & $\begin{array}{l}\text { Ajuda na locomoção pela cidade para o embarque e desembarque no transporte } \\
\text { publico. }\end{array}$ \\
\hline FFA_03 & Realiza a leitura de tela em sistemas informáticos. \\
\hline FFA_04 & Sistema operacional específico para deficientes visuais com audio descrição. \\
\hline FFA_05 & Ensino da linguagem Braille. \\
\hline FFA_06 & Avalia a acessibilidade dos locais públicos. \\
\hline FFA_07 & Jogo para o ensino de química em libras ou por estímulo de cores. \\
\hline FFA_08 & Ensino da linguagem de sinais (libras). \\
\hline FFA_09 & Sistema de sinalização para solicitar o embarque nas linhas de ônibus. \\
\hline FFA_10 & $\begin{array}{l}\text { Desenvolvimento de comunicação alternativa por pessoas não verbais ou que } \\
\text { apresentam alguma dificuldade de fala ou escrita. }\end{array}$ \\
\hline FFA_11 & Tradutor em legendas e audio descrição. \\
\hline FFA_12 & Simplificação léxica e sintática. \\
\hline
\end{tabular}

Fonte: Elaborado pelos autores (2019).

Baseado no mapeamento apresentado na Tabela 1 abaixo, observa-se que o aplicativo Hand Talk foi o mais citado entre os estudos - 10 dos EPs o cita (37,03\%), em seguida, o ProDeaf - 7 estudos (25,92\%). Outro dado que pode ser trabalhado a partir da Tabela 1 é sobre a quantidade de ferramentas e aplicativos para determinadas deficiências. Segundo a última Pesquisa Nacional de Saúde - PNS (IBGE, 2013), que é realizada a cada dez anos, a maior prevalência de deficientes no Brasil são de pessoas com deficiência visual - 3,6\% da população, em seguida vem a deficiência física com prevalência de $1,3 \%$, deficiência auditiva com $1,1 \%$ e deficiência intelectual com $0,8 \%$.

Baseado nestes dados, pode-se realizar uma comparação com as deficiências atendidas pelas ferramentas e aplicativos mapeados. Observa-se que a deficiência com maior número de indivíduos no país, a visual, foi a que obteve o maior número de ferramentas e aplicativos mapeados, 18 que representa $44 \%$ do total. A ordem de prevalência com a quantidade de ferramenta e aplicativos mapeados se mantém com relação a deficiência auditiva. No mapeamento foi a terceira deficiência com mais resultados mapeados, 7 ferramentas e aplicativos (17\% do total) e também mantém essa posição em relação ao predomínio na população brasileira. Para os indivíduos com algum tipo de deficit de aprendizagem, 20\% das ferramentas e aplicativos foram mapeados para eles, que totalizam 8 - a segunda deficiência com o maior resultado mapeado. Porém, de acordo com a PNS, ela é a deficiência com a menor quantidade de indivíduos no Brasil. Estes indivíduos são caracterizados com um quadro cognitivo abaixo da média esperada para atividades que envolvem compreensão e raciocínio. 
Como as ferramentas e aplicativos mapeados envolvem diferentes tipos de habilidades que serão estimuladas, um número elevado de resultados pode ser explicado sobre esta óptica.

Para a inclusão digital de indivíduos com alguma dificuldade de locomoção ou auditiva, a quantidade de ferramentas e aplicativos ficaram bem próximas, 6 (15\%) e 7 (17\%) respectivamente. Esta quantidade pode ser corroborada pelo número de pessoas com este tipo de deficiência no Brasil que possuem quantidade bem próximas. A menor quantidade de ferramentas e aplicativos mapeados foi para indivíduos com algum tipo de deficiência na fala, com apenas 5\% (2 ferramentas ou aplicativos). Na PNS não foram identificados indivíduos com este tipo de deficiência.

Tabela 1. Identificação das ferramentas e aplicativos.

\begin{tabular}{|c|c|c|c|c|c|}
\hline $\begin{array}{l}\text { IDENTIFICA- } \\
\text { DOR FFA }\end{array}$ & $\begin{array}{l}\text { FERRAMENTA/ } \\
\text { TÉCNICA/ } \\
\text { APLICATIVO }\end{array}$ & $\begin{array}{l}\text { EVIDÊNCIA } \\
\text { PRIMÁRIA }\end{array}$ & $\begin{array}{l}\text { CARACTE- } \\
\text { RISTICAS }\end{array}$ & $\begin{array}{c}\text { TIPO DE } \\
\text { TECNOLOGIA }\end{array}$ & $\begin{array}{l}\text { DEFICIÊNCIA } \\
\text { ATENDIDA }\end{array}$ \\
\hline EFA_01 & Be My Eyes & EP_01, EP_03 & FFA_01 & $\mathrm{AC}$ & Visual \\
\hline EFA_02 & Eye-D & EP_01 & FFA_01 & $\mathrm{AC}$ & Visual \\
\hline EFA_03 & Sonar & EP_01 & FFA_02 & $\mathrm{AC}$ & Visual \\
\hline EFT_04 & LianeTTS & EP_01 & FFA_03 & $\mathrm{AC}$ & Visual \\
\hline EFT_05 & Virtual Vision & EP_01 & FFA_03 & $\mathrm{AC}$ & Visual \\
\hline EFT_06 & Jaws & EP_01 & FFA_03 & $\mathrm{AC}$ & Visual \\
\hline EFT_07 & DOSVOX & $\begin{array}{l}\mathrm{EP}+01, \mathrm{EP} \_15, \\
\mathrm{EP}_{-}{ }_{23}\end{array}$ & FFA_04 & $\mathrm{AC}$ & Visual \\
\hline EFT_08 & Braille Play & EP_01, EP_11 & FFA_05 & $\mathrm{AC}$ & Visual \\
\hline EFT_09 & Braille Touch & EP_01, EP_11 & FFA_05 & $\mathrm{AC}$ & Visual \\
\hline EFT_10 & Guia de rodas & EP_05 & FFA_06 & $\mathrm{AC}$ & Locomoção \\
\hline EFT_11 & Q-LIBRAS & EP_02 & FFA_07 & $\mathrm{AC}$ & $\begin{array}{l}\text { Visual e } \\
\text { Auditiva }\end{array}$ \\
\hline EFT_12 & V-Libras & $\begin{array}{l}\mathrm{EP}+02, \mathrm{EP}_{-} 09, \\
\mathrm{EP}_{-}^{-13}\end{array}$ & FFA_08 & $\mathrm{AC}$ & Visual \\
\hline EFT_13 & ProDeaf & $\begin{array}{l}\mathrm{EP} \text { 02, EP_08, } \\
\mathrm{EP}^{-12} \mathrm{EP}_{-13}^{-13} \\
\mathrm{EP}^{-} 16, \mathrm{EP}_{-}^{-17}, \\
\mathrm{EP}_{-}^{-} 21\end{array}$ & FFA_08 & $\mathrm{AC}$ & $\begin{array}{l}\text { Auditiva e de } \\
\text { fala }\end{array}$ \\
\hline EFT_14 & Hand Talk & 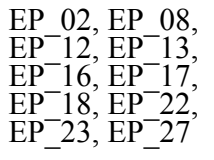 & FFA_08 & $\mathrm{AC}$ & $\begin{array}{l}\text { Auditiva e de } \\
\text { fala }\end{array}$ \\
\hline EFT_15 & DPS2000 & EP_03 & FFA_09 & $\mathrm{AC}$ & Visual \\
\hline EFT_16 & Busalert & EP_03, EP_15 & FFA_02 & $\mathrm{AC}$ & $\begin{array}{l}\text { Locomoção e } \\
\text { visual }\end{array}$ \\
\hline EFT_17 & $\begin{array}{l}\text { Smart Audio City } \\
\text { Guide }\end{array}$ & EP_03 & FFA_01 & $\mathrm{AC}$ & Visual \\
\hline EFT_18 & Wayfindr & EP_03 & FFA_01 & $\mathrm{AC}$ & Locomoção \\
\hline EFT_19 & LetMe Talk & $\begin{array}{l}\mathrm{EP} \text { 04, EP_12, } \\
\mathrm{EP}_{-}^{-19}\end{array}$ & FFA_10 & $\mathrm{AC}$ & $\begin{array}{l}\text { Dificuldade de } \\
\text { aprendizagem }\end{array}$ \\
\hline
\end{tabular}




\begin{tabular}{|c|c|c|c|c|c|}
\hline EFT_20 & SCALA & EP_04 & FFA_10 & $\mathrm{AC}$ & $\begin{array}{l}\text { Dificuldade de } \\
\text { aprendizagem }\end{array}$ \\
\hline EFT_21 & PictoDroid Lite & EP_04 & FFA_10 & $\mathrm{AC}$ & $\begin{array}{l}\text { Dificuldade de } \\
\text { aprendizagem }\end{array}$ \\
\hline EFT_22 & Vox4All & EP_04 & FFA_10 & $\mathrm{AC}$ & $\begin{array}{l}\text { Dificuldade de } \\
\text { aprendizagem }\end{array}$ \\
\hline EFT_23 & $\begin{array}{l}\text { Guia Turismo } \\
\text { Acessível }\end{array}$ & EP_05 & FFA_06 & $\mathrm{AC}$ & Locomoção \\
\hline EFT_24 & Wheelmap & EP_06 & FFA_06 & $\mathrm{AC}$ & Locomoção \\
\hline EFT_25 & Dá pra Ir?” & EP_06 & FFA_06 & $\mathrm{AC}$ & Locomoção \\
\hline EFT_26 & Linux acessível & EP_07 & FFA_04 & $\mathrm{AC}$ & Visual \\
\hline EFT_27 & Whatscine & EP_10, EP_15 & FFA_11 & $\mathrm{AC}$ & Visual e auditiva \\
\hline EFT_28 & MovieReading & $\mathrm{EP} \_10, \mathrm{EP}_{-} 15$ & FFA_11 & $\mathrm{AC}$ & Visual e auditiva \\
\hline EFT_29 & LIBRAS Game & EP_14 & FFA_08 & $\mathrm{AC}$ & Auditiva \\
\hline EFT_30 & EDUKITO & EP_20 & FFA_10 & D_EP & $\begin{array}{l}\text { Dificuldade de } \\
\text { aprendizagem }\end{array}$ \\
\hline EFT_31 & Multi-Trilhas & EP_21 & FFA_08 & $\mathrm{AC}$ & Auditiva \\
\hline EFT_32 & PorSimples & EP_24 & FFA_12 & D_EP & $\begin{array}{l}\text { Dificuldade de } \\
\text { aprendizagem }\end{array}$ \\
\hline EFT_33 & ABC Autismo & EP_25 & FFA_10 & $\mathrm{AC}$ & $\begin{array}{l}\text { Dificuldade de } \\
\text { aprendizagem }\end{array}$ \\
\hline EFT_34 & HangAut Game & $\mathrm{EP} \_25$ & FFA_10 & $\mathrm{AC}$ & $\begin{array}{l}\text { Dificuldade de } \\
\text { aprendizagem }\end{array}$ \\
\hline EFT_35 & CPqD Alcance & EP_26 & FFA_03 & D_EP & Visual \\
\hline
\end{tabular}

Fonte: Elaborada pelos autores (2019).

\section{Conclusão}

A contribuição que a utilização de ferramentas e aplicativos traz para a inclusão digital dos indivíduos e para a inserção das pessoas portadoras de algum tipo de deficiência, embora seja reconhecida, ainda necessita que haja uma maior difusão do conhecimento sobre quais estão disponíveis. O desafio encontrado na literatura é verificar através de métodos sistemáticos quais são essas ferramentas e aplicativos. Nesse sentido, o presente trabalho buscou investigar ferramentas e aplicativos que possam servir de apoio à inserção digital das pessoas com deficiência, sendo este o objetivo geral desta pesquisa.

O mapeamento sistemático desenvolvido teve o intuito de verificar, através de publicações acadêmicas, as ferramentas e aplicativos que podem ser utilizados na inclusão digital de pessoas com deficiência. Foi possível identificar uma quantidade relevante quanto às ferramentas e aplicativos, o que possibilita inferir sobre a importância da utilização desses meios para a inserção de pessoas no âmbito digital. No total foram 35 ferramentas e aplicativos mapeados para 5 diferentes deficiências. Porém, para a deficiência de fala obteve-se poucos resultados expressivos no mapeamento. Observa-se com o mapeamento um maior desenvolvimento de ferramentas e aplicativos que são voltados para pessoas com dificuldade de V. $18 \mathrm{~N}^{\mathrm{o}} 1$, julho, 2020 RENOTE DOI: 
aprendizagem e estes incorporam características que podem ajudar nas necessidades das pessoas com deficiência na fala.

Isto reflete também na relação das características presentes nas ferramentas e aplicativos. A extração permitiu observar que a característica "desenvolvimento de comunicação alternativa por pessoas não verbais ou que apresentam alguma dificuldade de fala ou escrita" com o identificador FFA_10 foi a mais presente entre as ferramentas e aplicativos mapeados, totalizando 7. Como essa característica pode englobar duas deficiências: a de fala e de aprendizagem, isto pode explicar esse número maior. As características "jogo para o ensino de química em libras ou por estímulo de cores", "sistema de sinalização para solicitar o embarque nas linhas de ônibus" e "simplificação léxica e sintática" apenas uma ferramenta ou aplicativo possui cada uma dessas características. Isto ocorre pois, são características muito específicas, ainda não amplamente disseminadas entre os desenvolvedores e na literatura. Dessa forma, este estudo permitiu conhecer as ferramentas e aplicativos que estão disponíveis e se mostram adequadas para a inclusão digital dos indivíduos portadores de algum tipo de deficiência. Esse conhecimento possibilita que este público alvo identifique a ferramenta ou aplicativo que mais se ajusta à sua realidade.

\section{Referências bibliográficas}

BRASIL. Lei $n^{\circ}$ 13.146, de 6 de julho de 2015. Institui a Lei Brasileira de Inclusão da Pessoa com Deficiência (Estatuto da Pessoa com Deficiência). Disponível em: http:// www.planalto.gov.br/ccivil_03/_ato2015-2018/2015/lei/113146.html. Acesso em: 4 jul. 2020.

CRUZ, R. O que as empresas podem fazer pela inclusão digital. São Paulo: Instituto Ethos, 2004.

IBGE - INSTITUTO BRASILEIRO DE GEOGRAFIA E ESTATÍSTICA. Pesquisa nacional de saúde, 2013.

KITCHENHAM, B.; CHARTERS, S. Guidelines for performing Systematic Literature Reviews in Software Engineering. Keele University. Durham, UK, 2007. MEDEIROS, L.D.; CARVALHO, M. M. Inclusão Digital como perspectiva positiva na sociedade da informação. Pesquisa Brasileira em Ciência da Informação e Biblioteconomia. Vol. 1; n.1. 2006.

NAZARENO, C.; BOCCHINO, E. V.; MENDES, F. L.; PAZ FILHO, J. S. Tecnologias da Informação e sociedade: o panorama brasileiro. Câmara dos deputados, coordenação de publicações, Brasília, 2006.

PETERSEN, K.; FELDT, R.; MUJTABA, S.; MATTSSON, M. Systematic Mapping Studies in Software Engineering. 12th International Conference on Evaluation and Assessment in Software Engineering (EASE). 2007, pp. 1-10.

VICTOR, S. L.; DRAGO, R.; CHICON, J. F. A educação inclusiva de crianças, adolescentes, jovens e adultos: avanços e desafios. Vitória: EDUFES, 2013. 